\title{
Editorials
}

\section{New concepts in iron deficiency anaemia}

Iron deficiency continues to be a major health issue worldwide - and iron deficiency anaemia is associated with welldocumented adverse health outcomes. ${ }^{1}$ Many studies have shown that iron deficiency is associated with a longer length of stay in hospital, ${ }^{2}$ cognitive dysfunction, ${ }^{3}$ increased risk of falls, ${ }^{4}$ and reduced life expectancy. ${ }^{5}$ Paradoxically, iron is one of the most abundant minerals on earth and is plentiful in all but the most restricted diets.

The discovery of a new peptide hormone, hepcidin, in 2001, which is made in the liver, is one key advance in this area. The discovery provides a critical insight into how iron deficiency arises in patients with inflammatory disease, despite an adequate intake of iron and no overt loss from bleeding. And, although previously parenteral iron therapy was hazardous and inefficient, modern formulations of intravenous iron overcome the perils of anaphylactic and anaphylactoid reactions ${ }^{6}$ caused by the dextran component used previously. Now, complete body iron replenishment can be administered feasibly in an ambulant daycase unit. With the advent of such powerful pharmaceutical tools, we have also begun to understand the more subtle aspects of iron deficiency, such as the remarkable effects of even sub-clinical iron deficiency, prior to the onset of anaemia, on cardiac and respiratory function. ${ }^{7-8}$

\section{HEPCIDIN - A REMARKABLE PEPTIDE HORMONE THAT BLOCKS IRON ABSORPTION}

It has long been recognised that anaemia arises in many chronic diseases, including chronic renal failure and inflammatory diseases such as rheumatoid arthritis, inflammatory bowel disease, and chronic osteomyelitis. Although loss oferythropoietin plays a role in advanced kidney diseases, in most other conditions the explanation for the anaemia of chronic disease has remained unsatisfactory. However, the new peptide hormone, hepcidin, has, it has been found, the remarkable property of causing profound iron deficiency in otherwise healthy individuals. ${ }^{\text {? }}$ This hormone is a small peptide, synthesised in the liver in response to inflammation anywhere in the body, and can profoundly limit the absorption of iron from the intestine. Total body iron stores are of the order of 2-3 $\mathrm{g}$. and are relatively stable in a healthy person.

\section{"[Hepcidin] can profoundly limit the absorption of iron}

from the intestine.

Inevitably, a small amount of iron is lost with cells being shed from the skin and intestine, and the amount, approximately $2 \mathrm{mg}$ per day, is replenished by absorption of that amount from diet. A normal diet contains an excess of iron and therefore most iron that is ingested passes through the intestine and is lost. In cases of iron deficiency, the absorption of iron can be increased to approximately $10 \mathrm{mg}$ per day and not more. When hepcidin levels are increased, for instance, in patients with an infection or other cause of inflammation, iron absorption is reduced below $2 \mathrm{mg}$ per day. Hence, under these circumstances, patients become more and more depleted of iron, particularly if there is also bleeding. such as in inflammatory bowel disease. In fact, hepcidin also prevents the release of iron from within macrophages, so that circulating iron levels drop even before total body stores are depleted. Thus, patients with inflammation can become anaemic quite rapidly

It is likely that this in-built mechanism causing iron deficiency and anaemia evolved in humans to help combat severe infections. There is some evidence that mild anaemia limits the severity of infections such as malaria, and it is also well established that bacteria absolutely require iron to survive and proliferate within the host organism. Although measuring hepcidin may be valuable, particularly in cases of resistant anaemia, a reliable clinical test has not yet been developed.

It is worth noting that serum ferritin, which is the most widely used and generally reliable measure of total body iron stores, can be misleading in patients with inflammatory diseases because it is also produced by the liver in increased amounts as a consequence of inflammation. Therefore, although simple iron deficiency such as that due to bleeding or inadequate diet is characterised by very low ferritin levels, the anaemia of chronic disease, which is also in effect due to a lack of iron available for erythropoiesis, may be associated with normal or even raised ferritin levels. In these cases, a useful clue is that standard inflammatory markers, such as $\mathrm{C}$-reactive protein (CRP) and erythrocyte sedimentation rate (ESR), are also usually elevated.

In most clinical circumstances, measuring iron status is made much more reliable by checking all of the following: haemoglobin $(\mathrm{Hb})$, mean corpuscular volume (MCV), ferritin, transferrin saturation (TSAT), and CRP or ESR to check for coexisting inflammation. A low ferritin and/or TSAT indicate iron deficiency regardless of any of the other values. And if the ferritin and CRP are low, one has to suspect that iron deficiency is present even if the $\mathrm{Hb}$ is normal, and this is even more likely if the TSAT is below $16 \%$.

\section{TREATMENT OF IRON DEFICIENCY — NOT JUST ORAL IRON}

Oral iron supplementation is generally cheap and, although not very well tolerated, usually associated only with non lifethreatening side effects such as abdominal pain, nausea, diarrhoea, and constipation. In contrast, older parenteral iron preparations, such as iron dextran, were notorious for being associated with potentially lethal anaphylactic and anaphylactoid reactions. ${ }^{2}$

However, as our understanding of the role of hepcidin in blocking the uptake of iron from the intestine has grown and it has become clearer that parenteral iron supplementation may, particularly in patients with inflammation, be preferred, there have been encouraging pharmaceutical developments producing intravenous iron replacement products that do not contain dextran. These can be administered in some cases in relatively high doses, such as $500 \mathrm{mg}$ to $1000 \mathrm{mg}$ in a single infusion, offering the opportunity to replace the total body deficit with one or two infusions. In some countries, for example, Switzerland, intravenous iron infusions, using newer preparations that lack dextran, are administered in primary care facilities within the community. In the UK at present, the Medicines and Healthcare 


\section{“... iron has physiological functions that are independent of its role in forming haemoglobin and red blood cells...}

products Regulatory Agency still requires that parenteral iron is administered in surroundings with access to facilities to treat severe infusion reactions, with the exception of patients with chronic kidney disease.

\section{IRON — THE HEART AND BEYOND}

Perhaps the most exciting news about iron in recent times is that what has long been known theoretically by the medical community, although neglected in practice - that iron has physiological functions that are independent of its role in forming haemoglobin and red blood cells - has been demonstrated dramatically in patients.? Iron, of course, is an essential component of cytochromes and other intracellular proteins and enzymes involved in producing cellular energy. Therefore, it would be expected that iron deficiency might affect neuronal and muscular function. And, indeed, in a group of patients with congestive cardiac failure and mild iron deficiency without overt anaemia, administering intravenous iron rapidly improved a number of markers of cardiac function, including a clinically significant increase in the capacity to exercise, as measured by the 6-minute walk test. ${ }^{7}$ In another field, administering iron reduced pulmonary artery hypertension caused by oxygen depletion, mimicking high altitude, and sequestering iron had the opposite effect.? These studies are being followed up and it would be both plausible - and unsurprising - if future evidence were to confirm that replenishing iron supplies in the body could benefit many complex functions of the body, including muscle strength and cognitive performance.

\section{Satish Keshav,}

Associate Professor, Translational Gastroenterology Unit, John Radcliffe Hospital, Oxford, Oxford University Hospitals NHS Foundation Trust and University of Oxford, Oxford.

\section{Richard Stevens,}

Chairman, Primary Care Society for Gastroenterology, London.

\section{Provenance}

Commissioned; externally peer reviewed.

\section{DOI: 10.3399/bjgp17X688465}

\section{ADDRESS FOR CORRESPONDENCE}

\section{Richard Stevens}

Primary Care Society for Gastroenterology, Satellite PR, 154-158 Shoreditch High Street, London

E1 6HU, UK.

E-mail: Richard.Stevenslathamesvalley.hee.nhs.uk

\section{REFERENCES}

1. Kassebaum NJ, Jasrasaria R, Naghavi M, et al. A systematic analysis of global anemia burden from 1990 to 2010. Blood 2014; 123(5): 615-624.

2. Clevenger B, Gurusamy $K$, Klein AA, et al. Systematic review and meta-analysis of iron therapy in anaemic adults without chronic kidney disease: updated and abridged Cochrane review. Eur J Heart Fail 2016; 18(7): 774-785.

3. Schneider $A L$, Jonassaint $C$, Sharrett $A R$, et al Hemoglobin, anemia, and cognitive function: the Atherosclerosis Risk in Communities study. J Gerontol A Biol Sci Med Sci2016. 71(6): 772-779.

4. Penninx BW, Pluijm SM, Lips P, et al. Late-life anaemia is associated with increased risk of recurrent falls. J Am Geriatr Soc 2005; 53(12): 2106-2111.

5. Shander A, Goodnough LT, Javidroozi M, et al. Iron deficiency anemia - bridging the knowledge and practice gap. Transfus Med Rev 2014; 28(3): 156-166

6. Rampton D, Folkersen J, Fishbane S, et al. Hypersensitivity reactions to intravenous iron: guidance for risk minimization and management. Haematologica 2014; 99(11): 1671-1676.

7. Anker SD, Comin Colet J, Filippatos G et al. FAIR-HF Trial Investigators. Ferric carboxymaltose in patients with heart failure and iron deficiency. N Engl J Med 2009: 361(25): 2436-2448.

8. Frise MC, Cheng HY, Nickol AH, et al. Clinical iron deficiency disturbs normal human responses to hypoxia. J Clin Invest 2016; 126(6): 2139-2150.

9. Nemeth E, Rivera S, Gabayan V, et al. IL-6 mediates hypoferremia of inflammation by inducing the synthesis of the iron regulatory hormone hepcidin. J Clin Invest 2004; 113(9): $1271-1276$ 